\title{
Go-with-the-flow: A Smartphone App for Realtime Tracking and Sonification of Physical Activity in CP
}

[Demo paper]

\author{
Joseph W. Newbold \\ UCL, London, UK \\ (joseph.newbold.14,
}

\author{
Aneesha Singh \\ UCL, London, UK \\ aneesha.singh.10,
}

\author{
Nadia Bianchi-Berthouze \\ UCL, London, UK \\ n.berthouze) @ucl.ac.uk
}

\begin{abstract}
Chronic pain is pain that persists past the expected time of healing. Unlike acute pain, chronic pain is often no longer a sign of damage and may never disappear. Remaining physically active is very important for people with chronic pain, but in the presence of such persistent pain it can be hard to maintain a good level of physical activity due to factors such as fear of pain or re-injury. This work demonstrates how sound feedback can be used to address some of the specific barriers to physical activity faced by people with CP. Using the Go-with-the-glow framework, sonifications are informed by physiotherapist and people with CP to help address the psychological needs and to help support movement and breathing during physical activity.
\end{abstract}

\section{Keywords}

Chronic pain, sonification, music

\section{INTRODUCTION}

Chronic pain (CP) is a condition which affects 7.8 million people in the UK costing the national health service about 12 billion pounds per annum [9]. CP is a persistent pain that remains after the expected time of healing [10] and results from changes in the nervous system that lead to pain amplification. Clinical resources cannot meet demand and many people do not get the help they need. Psychological factors such as fear and anxiety about activity exacerbating pain or damage restrict lives [11]. While technology has been used to monitor activity, it lacks the necessary psychological support. Technology modeled on other conditions such as stroke has also been developed for CP rehabilitation but ignores psychological factors particular to $\mathrm{CP}$.

\section{FRAMEWORK AND IMPLEMENTATION}

In this work, we present and evaluate a new sonification framework called Go-with-the-flow for $\mathrm{CP}$ rehabilitation $[8,7,5]$. The framework has been informed by studies with physiotherapists and people with CP and implemented through an app to track and give feedback on movement. The evaluation aimed to understand how the sonification of movement based on the Go-with-the-flow framework [7] facilitates both physical exercise and everyday functioning in people with CP. In addition the use of wearable respiration sensors, shown in Figure 1, for breathing sonification.
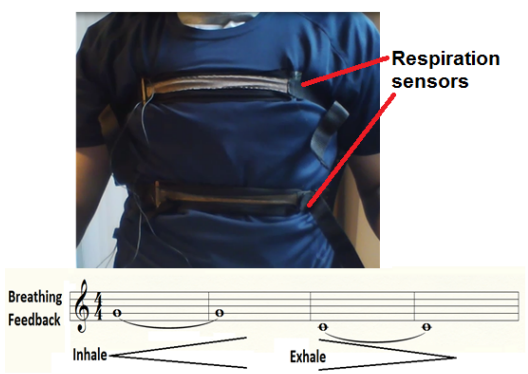

Figure 1: T-shirt based respiration sensors allow breathing to be tracked and a two note sonification is used to highlight the rhythm of a persons breathing, one note played on inhale and another on exhale [4]

The framework proposes articulation of user-defined sonified exercise spaces (SESs) tailored to psychological needs in relation to perceived physical capabilities. The system was implemented using an android-based smartphone, and the gyroscopes within the phone were used to measure movement. The SES was calibrated by the individual and divided into segments, as the individual movement through each segment the next note/chord in the sonification is triggered, as shown in Figure 2.

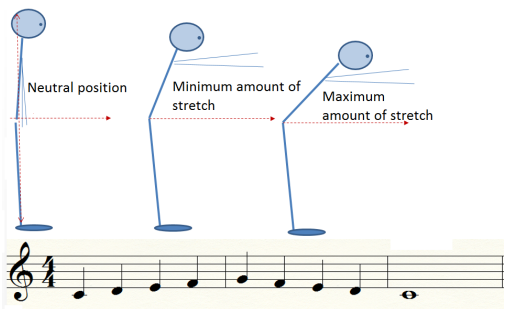

Figure 2: An exercise space is calibrated using a neutral position, comfortable amount of stretch and a maximum target stretch [4] 
The sonification was designed to address factors that emerged from the qualitative studies with people with chronic pain and physiotherapists:

Skills acquisition to take control of physical rehabilitation: Anchor points are calibrated through exploring a person's own body movement. To set an anchor point, the person performs a movement to the specific point and then stores it. The calibration phase is a body exploration phase, as done by physiotherapists. The app can be recalibrated any time, as needed, to address change in capability even for the same individual. Self-calibration can help people to discover physical capabilities and psychological needs, tailor activity to physical and psychological resources and gradually build capabilities.

Increase awareness of body movement to increase confidence in doing feared exercises: Sound is used to define an exercise space characterised by anchor points. These anchor points give information about body position with respect to the movement being executed and can be used by people to set targets. The feedback is designed to shift focus to an external pleasurable representation of the body as it moves rather than pain or feared movement.

Increase perceived value in moving to increase selfefficacy and reward: Sonification steps (e.g., distance between two notes) are calibrated to the psychological and physical capability of the person to do a movement. This ensures that even when people have restricted physical capability, small movements are still rewarded and they are motivated to continue the movement.

Increase awareness of protective movement: Protective behaviour may indicate increased anxiety towards the movement being performed or automaticity reached over time. Alterations to the sonification are implemented when the sensor detects protective behaviours. For example, adoption of an asymmetric position to avoid using a stiff or painful body part, alters the sound feedback to only play in one ear.

Promote progress and avoid over-activity: Overdoing can be an issue for people with CP that expect or desire normal mobility and especially on days of low pain will go too far, leading to setbacks. By using harmonic structures within the sound feedback, the sonification could be concluded musically and people can be invited to concluded their movement implicitly using peoples embodied relationship with music [2]. Conversely by using musical tension people can be persuaded to continue movement, allowing people to pass preconceived boundaries.

Focus on breathing and pacing: through peoples understanding of rhythm and their ability to synchronise their motor behaviours to it [6] [3] and the using of breathing sonification designed to highlight the rhythm of one's breathing, the rate of respiration can be manipulated [4]. For example to promote relaxation or to set the pacing of movement.

The app and framework were evaluated through a control study $(n=15)$, a focus group $(n=5)$, observation of people with CP using the device at home $(n=8)$ and a 2-week-use of the device by people with $\mathrm{CP}(\mathrm{n}=4)$. A further two control studies were conducted to evaluate how musical structure could be added to modify movement $(\mathrm{n}=17, \mathrm{n}=32)$.

\section{RESULTS}

Our studies indicated that sound feedback increased selfefficacy and confidence. Using their own body as a tool to set fine-grained goals/targets and tailoring feedback to self-calibrated psychological and physical capability and rewarding even small movements increased self-efficacy and perceived value of movement. It was also found that by using the idea of harmonic stability, people would be promoted to stop by a musically concluded sonification and would continue to move in a musical unresolved one. The evaluation showed that people reported increased perceived efficacy, control, motivation and pleasure in carrying out feared exercises when using the app. The results of the evaluation can be found in $[7,5]$. People found that the sonification framework tailored to specific psychological and physical needs increased self-efficacy, performance, and motivation.

\section{REFERENCES}

[1] E. Bigand. Perceiving musical stability: The effect of tonal structure, rhythm, and musical expertise. ... of Experimental Psychology: Human Perception and ..., 1997.

[2] M. Leman. Embodied music cognition and mediation technology. Mit Press, 2008.

[3] G. C. McIntosh, S. H. Brown, R. R. Rice, and M. H. Thaut. Rhythmic auditory-motor facilitation of gait patterns in patients with Parkinson's disease. Journal of Neurology, Neurosurgery \&5 Psychiatry, 1997.

[4] J. W. Newbold, N. Bianchi-Berthhouse, N. E. Gold, and A. Williams. Musically Informed Sonification for Self-Directed Chronic Pain Physical Rehabilitation. In Sound and Music Computing, 2015.

[5] J. W. Newbold, N. Bianchi-Berthouze, N. E. Gold, A. Tajadura-Jiménez, and A. Williams. Musically Informed Sonification for Chronic Pain Rehabili-tation: Facilitating Progress \& Avoiding Over-doing. In Proceedings of (ACM CHI 2016), San Jose, 2016.

[6] B. H. Repp. Sensorimotor synchronization: A review of the tapping literature. Psychonomic Bulletin 8 Review, 12(6):969-992, Dec. 2005.

[7] A. Singh, A. Klapper, J. Jia, A. Fidalgo, A. Tajadura-Jiménez, N. Kanakam, N. Bianchi-Berthouze, and A. Williams. Motivating People with Chronic Pain to Do Physical Activity: Opportunities for Technology Design. In Proceedings of (ACM CHI 2014), CHI '14, New York, 2014. ACM.

[8] A. Singh, D. Pollarolo, S. Piana, G. Volpe, A. Tajadura-Jimenez, G. Varni, H. Aung, I. Pouw, A. Williams, A. Camurri, and N. Berthouze. Go with the flow: Tracking, Analysis and Sonification of Movement and Breathing to Build Confidence in Activity Despite Chronic Pain. HCI, 30, 2015.

[9] The Health and Social Care Information Centre. Chronic pain [online] http://www.hscic.gov.uk/ catalogue/pub09300/hse2011-ch9-chronic-pain.pdf. 2011.

[10] D. C. Turk and T. E. ^Rudy. IASP taxonomy of chronic pain syndromes: preliminary assessment of reliability. Pain, 30(2):177-189, Aug. 1987.

[11] D. C. Turk and A. Okifuji. Psychological factors in chronic pain: evolution and revolution. Journal of consulting and clinical psychology, 70(3):678-690, 2002 . 